Revue

Revue de l'histoire des religions

de Ihistoire des religions

$3 \mid 2020$

Varia

Claude FLEURY, Les Mœurs des Israélites, édition critique par Volker KAPP

Paris, Honoré Champion (« Sources classiques », 136), 2018

Jacques Le Brun

OpenEdition

Journals

Édition électronique

URL : https://journals.openedition.org/rhr/10762

DOI : 10.4000/rhr.10762

ISSN : 2105-2573

Éditeur

Armand Colin

Édition imprimée

Date de publication : 1 septembre 2020

Pagination : 486-488

ISBN : 978-2-200-93327-2

ISSN : 0035-1423

Référence électronique

Jacques Le Brun, «Claude FleURY, Les Mœurs des Israélites, édition critique par Volker KaPp », Revue de I'histoire des religions [En ligne], 3 | 2020, mis en ligne le 01 janvier 2021, consulté le 06 janvier 2023. URL : http://journals.openedition.org/rhr/10762 ; DOI : https://doi.org/10.4000/rhr.10762

Ce document a été généré automatiquement le 6 janvier 2023

Tous droits réservés 


\section{Claude FLEURY, Les Mœurs des Israélites, édition critique par Volker KAPP}

Paris, Honoré Champion (« Sources classiques », 136), 2018

Jacques Le Brun

\section{RÉFÉRENCE}

Claude FleURY, Les Mcurs des Israélites, édition critique par Volker KAPP, Paris, Honoré Champion (« Sources classiques », 136), 2018, 356 p., $22 \mathrm{~cm}, 50 €$, ISBN

978-2-7453-4852-4.

1 De Claude Fleury, Noémie Hepp et Volker Kapp avaient donné chez le même éditeur en 2003 une édition critique d'Écrits de jeunesse. Volker Kapp publie aujourd'hui, avec une considérable introduction, une édition critique d'un des livres qui ont assuré à Fleury pendant deux siècles un grand écho auprès des historiens et du public, Les Mœurs des Israélites, dont la première édition, suivie de nombreuses autres, date de 1681 . C'est cette première édition qui sert de base à la présente publication, les nombreux passages et références bibliques ajoutés par Fleury lui-même en 1682 dans la seconde édition apparaissant in-texte en italiques. Un travail sur les intentions manifestées par l'auteur, développements nouveaux, addition de précisions ou de références, plutôt que corrections ou repentirs, est ainsi facilité. Dans son introduction, Volker Kapp a le mérite de replacer Les Mœurs des Israélites dans le cadre de toute la série des travaux historiques, juridiques, pédagogiques et rhétoriques de Fleury, et avant tout d'avoir mis en perspective l'œuvre symétrique, Les Mours des Chrétiens, de 1682, esquisse d'une comparaison, prolongement et complémentarité, travail à la fois historique et apologétique, les Chrétiens prenant la suite des Israélites et les renvoyant au rôle de précurseurs. Mais, loin de s'en tenir là, l'éditeur convoque les nombreux livres et opuscules de Fleury, portant sur le droit ecclésiastique, l'histoire ecclésiastique, le 
choix et la méthode des études, et sur la littérature de l'Antiquité. Et il s'appuie sur une vaste information; tout au plus aurait-il pu relever les jugements portés en 1681 dans leurs correspondances par certains érudits, expressément sur Les Mœurs des Israélites, par exemple Justel (Paris, Bibl. S.H.P.F. ms 811, p. 51) ou correspondance de Locke (Bodléienne C 12 fo $^{\circ} 96 \mathrm{v}^{\circ}$ ) ou les notes du P. Léonard (BnF, ms. fr. 22581 fo 47 ; fr. 19208 passim).

2 Tous les aspects littéraires du livre ainsi que les choix théologiques de Fleury sont bien mis en valeur par l'éditeur qui éclaire ce qui est dit des Israélites par ce qui est écrit dans ses autres œuvres sur Homère et sur l'Antiquité classique et patristique. Si Fleury, qui fut actif dans des groupes comme l'Académie Lamoignon ou le "Petit concile", animé par Bossuet et quelques autres, possédait une bonne culture historique (avec toutefois des réserves de la part d'érudits de son temps avec qui néanmoins il était en correspondance, voir le Longueruana, II, p. 110-111 et BnF ms. fr 9519 p. 11, 37), cette culture, dans sa distance par rapport à la théologie scolastique et son orientation juridique, ne prenait pas en compte les travaux des érudits les plus novateurs; ainsi la perspective philologique et l'élaboration d'une histoire du texte par Richard Simon, malgré les développements biaisés d'un Jean Le Clerc (p. 83), lui étaient étrangères. Une formule de Jean-Louis Quantin, rapportée par V. Kapp, caractérise bien l'œuvre savante de Fleury, une « historiographie gallicane » (p. 38). Cela explique peut-être l'histoire de ces livres de Fleury, leur considérable succès jusqu'au cours du $\mathrm{xIX}^{\mathrm{e}}$ siècle puis leur caractère irrémédiablement dépassé. On peut voir dans ces œuvres de Fleury une bonne anticipation de ce que les historiens étudieront à propos $d u \mathrm{XIX}^{\mathrm{e}}$ siècle sous le nom de "science catholique», science jusqu'à un certain point, mais «entre apologétique et vulgarisation » (Claude Langlois et François Laplanche dir., La science catholique, Paris, Cerf, 1992). On peut penser que si Fleury avait accepté la proposition faite par le cardinal de Noailles de donner une traduction en français du Nouveau Testament (BnF ms. fr. $19208 \mathrm{f}^{\circ} 131$ et $^{\circ}$ ), il se serait contenté de traduire la Vulgate (p. 70). Le catalogue de sa bibliothèque et les dates de ses achats de livres, conservés dans les manuscrits fr. 9517-9521 de la BnF, ne feront que confirmer notre impression concernant les caractères et les limites de son érudition.

3 C'est toutefois pour d'autres raisons que Les Mœurs des Israélites peuvent retenir aujourd'hui l'attention des historiens. Il faudra pour cela décentrer notre mode de lecture et poser au texte de Fleury des questions qui n'auront pu l'être qu'avec le renouvellement de la science historique. Car l'œuvre de Fleury exprimait avant tout une conception du monde, celle d'un monde dont la «morale » était une perspective fondamentale, «la vraie religion » étant «le fondement de la morale» (p.118, répété presque textuellement p. 202). Il s'agissait donc pour les Israélites, non pas de vaincre la nature, perspective des moralistes, point seulement rigoristes, mais de mener une vie "conforme à la nature "; c'est Bossuet qui par ces mots résumait le livre dont il écrivait l'approbation (p.11), mais Fleury les reprenait dès les premières lignes de son livre : « la vie humaine la plus conforme à la nature » (p. 117). Et il pouvait soutenir que la vie des Israélites " a été plus heureuse, parce qu'elle était plus naturelle» (p.136). Bien plus, selon lui, les lois de Moïse pouvaient avoir été révélées par Dieu, elles avaient néanmoins "des raisons naturelles" (p. 158 et aussi p.185). Impossible de ne pas replacer ces affirmations de Fleury dans le mouvement de renouvellement de l'idée de nature qui conduira à la philosophie des Lumières et que les travaux de Jean Ehrard, de Bernard Tocanne et de Roger Mercier ont naguère analysé. Autre axe de la pensée de 
Fleury qu'il convient de souligner, le recours à la raison, non pas la raison des théologiens et des philosophes, jusqu'à Fénelon, qui voyaient en Dieu son origine et son garant, mais la raison au sens de ce qui est «raisonnable». Ainsi, lorsque Fleury demandait de ne juger les mœurs des Israélites «que par le bon sens et par la droite raison » (p. 118), il donnait à ces mots un sens qui annonce de prochaines mutations.

Il en est de même pour une notion qui revient en mainte occasion dans Les Mœurs des Israélites, l'utilité. Les préceptes rituels de la loi de Moïse s'expliquent par leur utilité (p. 158-160), ce qui ne leur donne aucun caractère «nouveau » ni «extraordinaire ». Les traditions que transmettait le Pentateuque étaient "nobles» et "utiles " et excitaient à la « vertu » (p.173), et les seules études auxquelles se livraient les Israélites étaient des études « vraiment utiles » (p. 177). Nous savons après les travaux de Michel Foucault (dans Les mots et les choses) et ceux de Michel de Certeau (dans L'écriture de l'histoire) combien la pensée des Lumières jusqu'à Hegel était marquée par l'utilité, l'utilité étant l'étalon auquel juger des croyances religieuses. La lecture de Fleury nous montre que les origines de cette pensée remontent au christianisme du XVII ${ }^{\mathrm{e}}$ siècle, la crise de la mystique au temps de Fénelon s'inscrivant dans cette histoire. Il sera peu étonnant de voir ce dernier juger Fleury « très faible théologien » (Revue Bossuet, 1909, p. 70). Mais Fleury semble assumer parfaitement la mutation intellectuelle dont il est à la fois le témoin et l'acteur : il n'hésitait pas à écrire : «Je ne vois point ici la nécessité de recourir au miracle et à la révélation " pour expliquer «la mémoire des choses passées » et il se contentait d'invoquer ce qui était "plus vraisemblable» (p. 120-121). Les mariages chez les Israélites, étaient « un contrat civil » sans « aucune cérémonie de religion» (p. 166); l'inceste, la polygamie ne furent interdits qu'ultérieurement par Dieu, et la virginité peu reconnue (p. 166-169) ; quant aux funérailles, c'étaient « action profane » sans "aucune cérémonie de religion » (p. 186). Que restait-il du peuple de l'Ancien Testament?

Si la nature était le guide de sa vie la comparaison avec les peuples primitifs découverts en ces derniers siècles s'imposait, et Fleury ne manque pas d'esquisser, timidement sans doute, des perspectives comparatistes en référence à des peuples étrangers au peuple de l'Ancien Testament: les peuples de l'Amérique (p.174), ceux des Indes et «d'autres pays éloignés » (p. 200), « les Nègres, les Caraïbes et les Iroquois » (p. 273) sont-ils vraiment différents de ces Israélites dont on relate les mœurs? Ce sont sans doute ces vues, par lesquelles Fleury involontairement annonçait l'avenir, qui font l'importance des Moeurs des Israélites, Arnold Van Gennep (cité p. 91) l'avait reconnu dans un pertinent article de 1920 (Revue de l'histoire des religions, 82, 1920, p. 139-162) et l'édition critique que nous donne Volker Kapp nous aidera à affronter cette question brûlante pour l'historien : comment du nouveau s'introduit dans la pensée?

\section{AUTEURS}

JACQUES LE BRUN

École pratique des Hautes Études, Paris. 